\title{
Biochemical markers of bone turnover in Croatian Warmblood horses undergoing medium level show jumping exercise
}

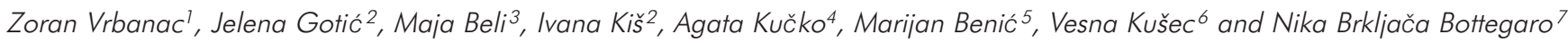 \\ 1 Department of Radiology, Ultrasound Diagnostic and Physical Therapy, Faculty of Veterinary Medicine, University of Zagreb, Zagreb, Croatia \\ ${ }^{2}$ Clinic for Internal Diseases, Faculty of Veterinary Medicine, University of Zagreb, Zagreb, Croatia \\ ${ }^{3}$ Department of Pathophysiology, Faculty of Veterinary Medicine, University of Zagreb, Zagreb, Croatia \\ ${ }^{4}$ Faculty of Veterinary Medicine, University of Zagreb, Zagreb, Croatia, student \\ 7 Surgery, Orthopaedics and Ophthalmology Clinic, Faculty of Veterinary Medicine, University of Zagreb, Zagreb, Croatia \\ ${ }^{6}$ Clinical Institute of Laboratory Diagnosis, Faculty of Veterinary Medicine, University of Zagreb, Zagreb, Croatia \\ 7 Clinical Hospital Centre, Zagreb, Croatia
}

\begin{abstract}
Summary: Bone turnover is a lifelong process during which the bone tissue undergoes processes that are physiologically in balance, formation and resorption. The aim of this study was to establish a profile of serum concentrations of biomarkers of bone turnover in Croatian Warmblood horses. Blood samples from 28 healthy horses used for medium level show jumping exercise were used in this study. Horses were trained for an average of six days per week with standard exercise of walking, trotting, cantering and jumping. Blood sampling was performed at the same time of the day with an aim to reduce diurnal variations. The samples were processed using commercially available human assays, validated for use in horses, for evaluation of bone alkaline phosphatase (BALP), osteocalcin (OC) and carboxy-terminal cross-linked telopeptide of type I collagen (CTX). The effects of age and gender on biomarker concentration were analysed. The concentrations of BALP and CTX peaked in youngest horses and all biomarkers showed a progressive decline with age. Gender difference in biomarker concentration was noted only for CTX with male horses having higher values. There was no statistically significant correlation between studied biomarkers, although their similar trends suggested that serum levels of bone resorption markers follow the same evolution as bone formation markers. The results of this study indicate that during the first years of show jumping training there is still pronounced bone turnover.
\end{abstract}

Keywords: bone alkaline phosphatase, bone turnover, carboxy-terminal cross-linked telopeptide of type I collagen, equine, osteocalcin, Warmblood horse

\begin{abstract}
Citation: Vrbanac Z., Gotić J., Beli M., Kiš I., Kučko A., Benić M., Kušec V., Brkljača Bottegaro N. (2017) Biochemical markers of bone turnover in Croatian Warmblood horses undergoing medium level show jumping exercise. Pferdeheilkunde 33, 371-376; DOI 10.21836/PEM20170407
\end{abstract}

Correspondence: Jelena Gotić, DVM, PhD, Clinic for Internal Diseases, Faculty of Veterinary Medicine, University of Zagreb, Heinzelova 55, Zagreb Croatia, Email: jelena.gotic@vef.hr

\section{Introduction}

Bone turnover is a lifelong process during which the bone tissue undergoes two main processes that are physiologically in balance, formation and resorption. The rate of bone turnover can be assessed by measuring the biochemical markers of bone turnover (Allen 2003). These markers are the bone cells' i.e. osteoblasts' and osteoclasts' products which are secreted into the circulation during formation and resorption of the bone. Concentration of the markers can be measured in serum and urine by different immunoenzyme (ELISA) or radioimmune (RIA) assays (Allen et al. 2000).

Among many, two commonly used markers that reflect bone formation are bone alkaline phosphatase (BALP) and osteocalcin $(O C)$, which are synthesised by osteoblasts. Presumably BALP has a role in bone matrix calcification, though its precise function in the formation process remains unknown. Bone alkaline phosphatase is a cell-membrane-associated ectoenzyme and in horses has been localized on the surface membrane of chondrocytes with concentration of activity on the matrix vesicles on the growth plate (Henson et al. 1995, Lepage et al. 2001).
Osteocalcin $(\mathrm{OC})$ is the most abundant noncollagenous protein that for 70-90\% binds to hydroxyapatite crystals in bone matrix, while the smaller portion of produced $O C$ is released in the blood and excreted by kidneys (Allen 2003). The exact biologic function of $O C$ is still not completely understood, but OC is accepted as an indicator of osteoblast activity (Risteli and Risteli 1993).

The major physiological determinant of BALP and OC concentrations is age. Inverse correlation between age and BALP and $O C$ concentrations has been noted with a significant slowdown in the rate of bone formation in adults compared to foals (Lepage et al. 1990). Gender has been shown to have influence on BALP and OC serum concentration in horses, but only when they are actively exercised (Lepage et al. 1992, Fletcher et al. 1998, Jackson et al. 2003a).

During bone resorption, osteoclasts break down bone tissue and collagen fragments are released into the blood and excreted by urine. Fragments released from the amino and carboxy terminal sites of collagen type I are called $\mathrm{N}$-terminal cross-linked telopeptide of type I collagen (NTX) and C-termi- 
nal cross-linked telopeptide of type I collagen (CTX) (Seibel 2005). Both telopeptides can be measured in blood or urine as bone resorption markers.

Bone markers provide a simple, rapid, non-invasive way of investigating bone metabolism in horses (Black et al. 1999) and can potentially be applied for early diagnosis of skeletal pathology. Furthermore, biomarkers can be used in order to determine responses to treatment and for monitoring optimisation of exercise regimens in terms of skeletal adaptive responses.

Influence on marker expression is complex (age, exercise, disease, surgery or medical treatment) and makes differentiation between metabolic bone diseases or bone adaptation to exercise impossible. It is therefore advised to use a panel of biomarkers of formation and resorption whenever possible (Price et al. 1995, Lepage et al. 2001).

Most of the studies on bone biomarker changes have been carried out on racehorses (Lepage et al. 1992, Price et al. 2001, Jackson et al. 2003a, Jackson et al. 2015) and the lack of information on changes in bone metabolism in show jumping horses has led us to perform this study. Show jumping horses are of interest when interpreting bone metabolism changes over longer period of time in contrast to racehorses, since racehorses' sports careers are evidently shorter, and make it impossible to evaluate bone marker changes in older horses in training. We hypothesised that biomarker level during active training can be influenced by gender and age. The aim of this study was to establish a profile of serum concentrations BALP, OC and CTX in Croatian Warmblood horses of different gender and ages in training.

\section{Material and methods}

Animals

Twenty-eight Croatian Warmblood horses; 15 mares and 13 geldings were included in this study. The mean $( \pm S D)$ age of horses was $8.3 \pm 3.6(4-16)$ years. The horses were classified in groups according to gender and age (see Tab. 1). All horses were healthy, with no history of lameness needing veterinary assistance in the last 2 years. This experimental group was regularly exercised on a medium level, and none of the horses has ever been used for breeding. All horses belonged to the same stable, and were kept in the same conditions, fed the same concentrate ration and hay. Water was provided ad libitum via automatic dispensers.

\section{Exercise}

Horses were trained for an average of six days per week with standard exercise of walking, trotting, cantering and jumping. They had jumping trainings up to two days weekly with jumps heightening at most $1.30 \mathrm{~m}$. All the horses included in the study had specific individual training programs in accordance with their training level.

\section{Blood sampling}

Blood sampling was conducted as a part of the annual veterinary physical examination requested by the horses' owners and was done by the authors. Written consent to use the results of the blood sampling for scientific purposes was given to the authors. Before the sampling, the horses were not exercised for one day. Blood samples were taken on the same day, within 30 minutes time frame in the morning between 7:00 and 7:30 a.m. Blood was obtained from the jugular vein in vacutainer tubes and placed on ice. Within one hour from collection, the blood was centrifuged at $3500 \mathrm{~g}$ for $15 \mathrm{~min}$ at $4^{\circ} \mathrm{C}$. Upon centrifugation aliquot serum samples were frozen at $-80^{\circ} \mathrm{C}$ until laboratory analyses.

\section{Assays}

The activity of BALP was measured using a commercial immunoassay kit (MetraTM BAP EIA kit, Quidel Corp., San Diego, CA, USA). The concentration of $\bigcirc \mathrm{C}$ was determined by using a commercial kit (Metra Osteocalcin, Quidel Corp., San Diego, CA, USA). The concentration of CTX was determined by using a commercial kit (Serum CrossLaps ELISA, Nordic Bioscience Diagnostics, Denmark). All measurements were performed according to manufacturer's instructions. The assays used had been previously validated for use with equine serum and proved to have good cross-reactivity with equine BALP, OC and CTX (Hoyt and Siciliano 1999, Filipović et al. 2014, Fueller et al. 2001, Billinghurst et al. 2003).

\begin{tabular}{|c|c|c|c|c|c|c|c|c|}
\hline Group & female & male & mean & $\min$ & $\max$ & median & SD & SE \\
\hline 1. $\leq 5$ years & 4.00 & 4.00 & 4.37 & 4.00 & 5.00 & 4.00 & 0.51 & 0.18 \\
\hline 2. 6 to 9 years & 5.00 & 6.00 & 7.63 & 6.00 & 9.00 & 8.00 & 0.92 & 0.27 \\
\hline 3. $\geq 10$ years & 4.00 & 5.00 & 12.88 & 10.00 & 16.00 & 13.00 & 1.76 & 0.58 \\
\hline
\end{tabular}

min - minimal measured value; max - maximal measured value; SD - standard deviation; SE - standard error

Table 2 Values of BALP, OC and CTX in serum samples from the study population of 28 Croatian Warmblood horses

\begin{tabular}{|c|c|c|c|c|c|c|}
\hline Parameters & mean & $\min$ & $\max$ & median & SD & SE \\
\hline BALP U/L & 33.80 & 18.00 & 48.20 & 31.80 & 7.26 & 1.37 \\
\hline$O C \mu \mathrm{g} / \mathrm{L}$ & 28.57 & 2.00 & 57.00 & 26.50 & 16.87 & 3.19 \\
\hline CTX $\mu \mathrm{g} / \mathrm{L}$ & 0.18 & 0.08 & 0.41 & 0.17 & 0.08 & 0.01 \\
\hline
\end{tabular}

min - minimal measured value: max - maximal measured value; SD - standard deviation; SE - standard error; BALP - bone alkaline phosphatase;

$\mathrm{OC}$ - osteocalcin; CTX - carboxy-terminal cross-linked telopeptide of type I collagen 
Statistical analysis

MedCalc software version 12.5.0.0. for Windows (MedCalc Software bvba, Ostend, Belgium) was used for statistical analysis. Continuous variables were tested for normality with the Kolmogorov-Smirnov test. Differences in biomarker levels between age and gender groups were assessed using oneway ANOVA and Student-Newman-Keuls post-hoc test for all pairwise comparisons. Comparison between gender groups was performed by t-test. Association of biomarkers with age was assessed using Pearson's correlation coefficient. Level of statistical significance was set at $p<0.05$.
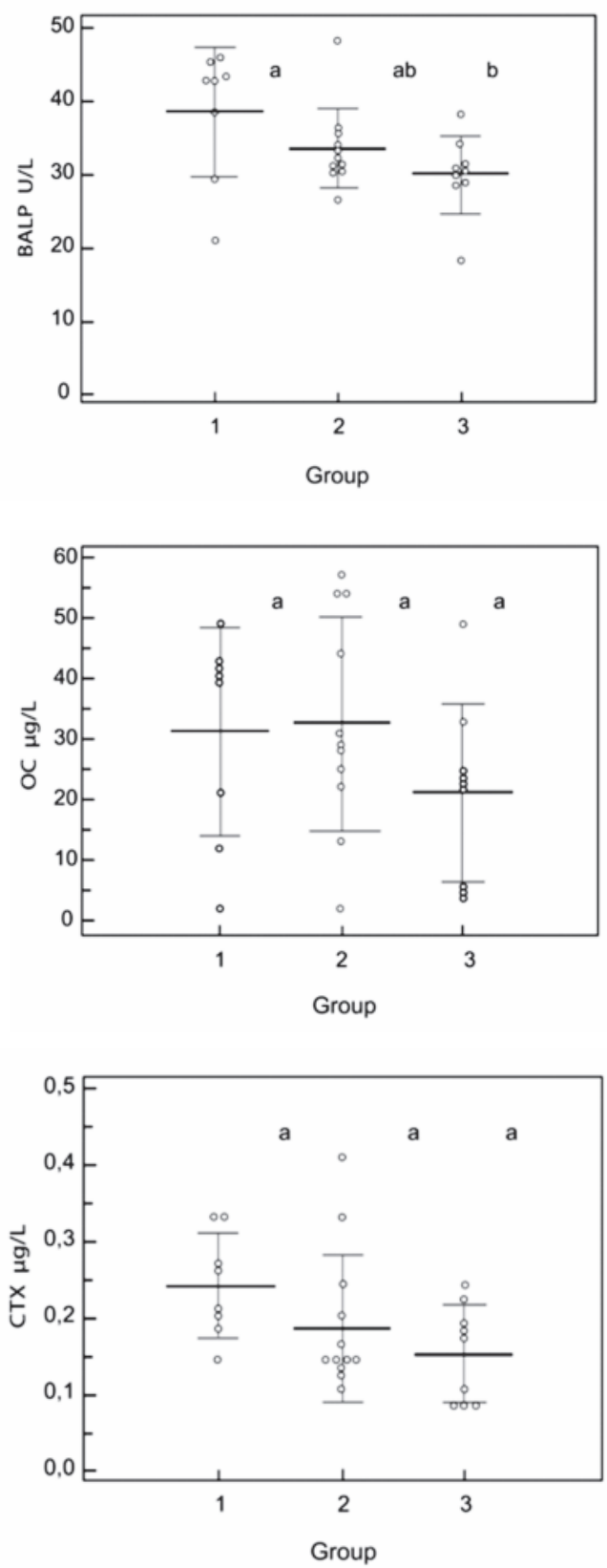

Fig. 1 Values of serum bone markers concentration, bone alkaline phosphatase (BALP), osteocalcin (OC) and carboxy-terminal cross-linked telopeptide of type I collagen (CTX), in serum samples from the study population of 28 Croatian Warmblood horses among three age groups. Dots represent individual values, horizontal bar and markers represent mean and standard deviation. Different letters $(a, b)$ indicate a significant difference $(p=0.044)$.

\section{Results}

Serum concentrations of OC and CTX and the activity of BALP of all tested horses are presented in Table 2. The bone marker values per age class are presented in Figure 1.

The mean $( \pm$ SD) BALP activity in the first group was 38.55 $( \pm 8.98) \mathrm{U} / \mathrm{L}$, in the second group $33.5( \pm 5.57) \mathrm{U} / \mathrm{L}$ and in the third group $29.95( \pm 5.42) \mathrm{U} / \mathrm{L}$. The highest BALP activity was $48.2 \mathrm{U} / \mathrm{L}$ in the second age group (horses between six and nine years of age). The lowest BALP activity was $18 \mathrm{U} / \mathrm{L}$, recorded in the youngest group (horses younger than five years of age). There was a significant difference between the first and the third group $(p=0.044)$. The mean $( \pm S D) \bigcirc C$ concentration in the youngest group was 31.25 $( \pm 17.2) \mu \mathrm{g} / \mathrm{L}$, in the second age group $32.63( \pm 17.8) \mu \mathrm{g} / \mathrm{L}$ and $21.22( \pm 14.7) \mu \mathrm{g} / \mathrm{L}$ in the oldest horses. No significant differences were found between groups. The mean $( \pm S D)$ CTX concentration in the youngest group was 0.24 $( \pm 0.06) \mu \mathrm{g} / \mathrm{L}$, in the second group $0.18( \pm 0.09) \mu \mathrm{g} / \mathrm{L}$ and in the oldest $0.14( \pm 0.06) \mu \mathrm{g} / \mathrm{L}$. The carboxy-terminal cross-linked telopeptide of type I collagen concentration had a decreasing tendency from the first to the third age group, although not statistically significant.

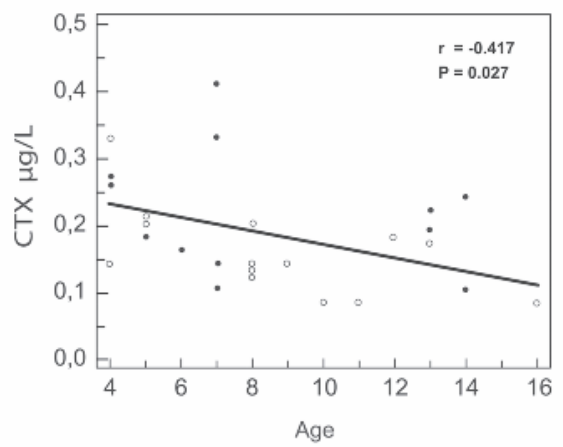

Fig. 2 Correlation between age and carboxy-terminal cross-linked telopeptide of type I collagen (CTX) serum concentration in a population of Croatian Warmblood horses. The correlation between age and CTX values was negative $(r=-0.417, p=0.027)$. The blank dots represent mares, the filled dots represent geldings.

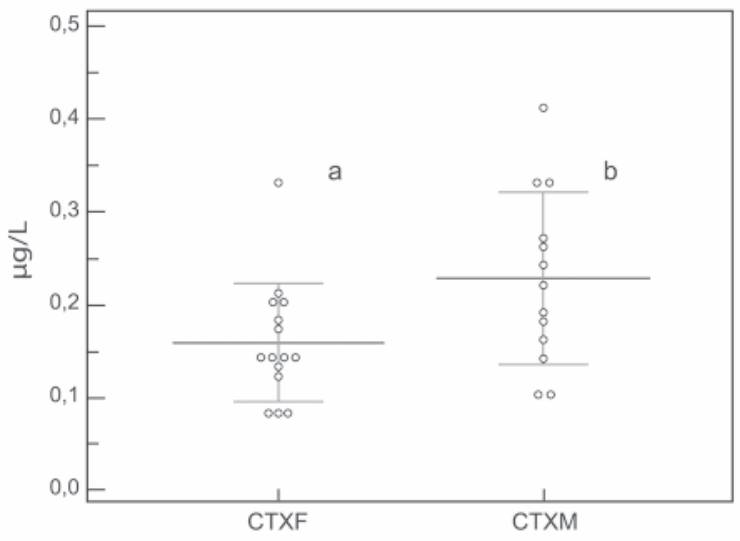

Fig. 3 Values of serum carboxy-terminal cross-linked telopeptide of type I collagen (CTX) concentration in female (CTXF) and male (CTXM) groups. Dots represent individual values, horizontal bar and markers represent mean and standard deviation. Different letters $(a, b)$ indicate a significant difference $(p=0.035)$. 
Whereas the relations between bone metabolism parameters and age show a decreasing tendency for all parameters, but only the correlation of CTX with age was significant $(r=-0.417, p=0.027)$, as shown in Figure 2. Gender difference in biomarker concentration in the overall sample was significant only for CTX with male horses having higher values $(p=0.035)$ (see Fig. 3).

\section{Discussion}

Exercise is known to increase bone mineral density, thereby enhancing bone mechanical strength with decreasing bone turnover (Vervuert et al. 2002, Jackson et al. 2003b, RoshanGhias et al. 2011 ). The current study is to establish bone turnover biomarkers concentration in Croatian Warmblood horses subjected to medium-level jump training. The age and/or gender influences on bone turnover could be demonstrated in some of these markers.

The $\mathrm{OC}$ values in this study were slightly higher in the second age group but showed a decrease in the oldest group of horses, although the difference was not statistically significant. Those results just partially coincide with the findings of Carstjanen et al. (2003), since they recorded the highest OC values in the horses younger than five years of age. The difference could possibly be explained by the fact that we did not use very young animals, but horses already in training from four years of age. Skeletal growth in young animals is characterized by a rapid rate of bone turnover, with predominance of bone formation (Black et al. 1999). Pastoret et al. (2007) noted the most pronounced decrease in OC values between birth and one year of age in Ardenner horses. In the current study there were no horses younger than four years of age, since the aim was to establish biomarker levels in horses in training. Black et al. (1999) in their study noticed three times higher $\mathrm{OC}$ values in weanlings compared to adult horses. Similar results were also reported by Lepage et al. (1992), who noted a significant inverse correlation of age and serum $\mathrm{OC}$ levels in Standardbred horses from birth to five years of age, while Vervuert et al. (2005) found a decrease in plasma $\mathrm{OC}$ at the beginning of the training period and assumed it might increase the risk for skeletal injuries during the early stage of training. In the present study no significant correlation between $\mathrm{OC}$ values and age was recorded, but, as said earlier, this may be explained by the ages of he selected horses.

Increased levels of CTX indicate increased bone collagen degradation since type I collagen accounts for $90 \%$ of the organic bone matrix (Billinghurst et al. 2003). The results concerning CTX followed the expected trend with age, as there was a significant correlation between CTX values and age, with younger horses having higher values of the biomarker. Those results seem to be in contrast with the findings of Billinghurst et al. (2003), who recorded an increase in CTX values with age until nine months of age. However, their population was very different, as they had included only horses in the first year of life, featuring intensive skeletal growth and modelling. Pastoret et al. (2007) found no correlation of age with the concentration of CTX in a study in Ardenner horses and similar findings were reported by Filipović et al. (2014).
A significant difference between the values of the youngest and the oldest group of horses demonstrated the influence of age on serum BALP concentrations. Horses in the middle age group also showed lower values of serum BALP in comparison with the youngest group of horses, but this difference was not statistically significant. Price et al. (1995) demonstrated a decrease with age in serum BALP. The same study also reports total ALP (alkaline phosphatase) levels decreasing with age, but since it did not correlate significantly with BALP in older animals it indicated that total ALP is not useful for detecting small changes in osteoblast activity in adult horses. Up to $92 \%$ of total serum ALP in newborn foals is attributed to BALP compared to around $20 \%$ in adult animals (Price et al. 1995), which is comparable to humans. In young dogs BALP is also age-related and seen as a result of high basal bone turnover rates in the growing, skeletally immature animals (Saini and Saini 1978). Once the animal reaches skeletal maturity there is a sharp decline in marker secretion (Price et al. 1995). Higher concentrations of various biochemical markers of bone resorption and formation in young horses in comparison to adult animals indicate greater bone modelling or remodelling, also described in humans (Carstanjen et al. 2004).

There was a gender difference in CTX concentration with higher values in male horses, which finding is consistent with the results of Jackson et al. (2003a). Higher CTX values in colts were in that study attributed to earlier skeletal maturation and smaller bone size in fillies, although our results suggest this effect persists also after cessation of growth. Gender differences were not noted for $\mathrm{OC}$ what could be attributed to the limited concentration of $\mathrm{OC}$ in the adult age. Contraire results were noted by Lepage et al. (1992) where exercised fillies showed higher serum $\mathrm{OC}$ values than geldings possibly caused by higher peripheral steroid hormone concentrations in females, when compared to castrated males (Fletcher et al. 2000). There was also no significant correlation between BALP and gender of the tested animals.

Blood sampling was performed at the same moment of the day to reduce variation due to diurnal variation. Significant $24 \mathrm{~h}$ variations in $\mathrm{OC}$ concentrations were found in adult geldings (Black et al. 1999) and young Thoroughbred mares (Jackson et al. 2003c). Sampling in the present study was done in September, as increases of serum OC levels have been noted in Thoroughbreds in October (Price et al. 2001), as well as in November in Ardenner horses (Pastoret et al. 2007).

In the present study, the correlation between the biomarkers was low, but the results confirm that serum levels of bone resorption markers followed the same trend as those of bone formation markers.

The small number of subjects in the present cohort could be considered a limitation of this study. However, the aim was to study bone turnover biomarkers in healthy show jumping horses of the same breed subjected to the same training regime, which limited the number of available horses. Future studies should include gender and age-matched control subjects without training and include longitudinal studies of selected biomarkers, possibly reflecting the training effect of bone turnover biomarkers. 


\section{Conclusion}

The results of this study support the observation of higher bone turnover in younger show jumping horses in training, especially during their first years of competition. Gender differences noted in this study showed higher values for CTX in male horses, and this effect persisted with age. These results are a contribution to the study of bone metabolism in veterinary medicine in show jumping horses.

\section{Animal Welfare Statement}

The use of animals in the present study under the ethical and animal welfare standards was approved by the Ethics Committee of the Faculty of Veterinary Medicine University of Zagreb (File no.: 640-01/16-17/25, Record no.: 251-6101/139-16-2)."

\section{Informed consent statement}

The authors confirm that all animal owners have been informed and signed consent that data concerning their horses may be used as a part of a scientific publication."

\section{References}

Allen L. C. V., Allen M. J., Breur G. J., Hoffmann W. E., Richardson D. C. (2000) A comparison of two techniques for the determination of serum bone-specific alkaline phosphatase activity in dogs. Res. Vet. Sci. 68, 231-235

Allen M. J. (2003) Biochemical markers of bone metabolism in animals: uses and limitations. Vet. Clin. Pathol. 32, 101-113

Billinghurst R. C., Brama P. A., van Weeren P. R., Knowlton M. S., Mcilwraith C. W. (2003) Significant exercise-related changes in the serum levels of two biomarkers of collagen metabolism in young horses. Osteoarth. Cartilage 11, 760-769

Black A., Schoknecht P. A., Ralston S. L., Shapses S. A. (1999) Diurnal variation and age differences in the biochemical markers of bone turnover in horses. J. Anim. Sci. 77, 75-83

Carstanjen B., Hoyle N. R., Gabriel A., Hars O., Sandersen C., Amory H., Remy B. (2004) Evaluation of plasma carboxy-terminal cross- linking telopeptide of type I collagen concentration in horses. Am. J. Vet. Res. 65, 104-109

Filipović N., Stojević Z. Plevnik N., Mašek T., Prvanović N., Tušek Z. (2014) The influence of age on bone metabolism in mares during late pregnancy and lactation. Res. Vet. Sci. 97, 194-198

Fletcher K. L., Topliff D. R., Freeman D. W., Cooper S. R., Looper M. L. (1998) Effects of sex and weaning on serum osteocalcin concentration in horses. Animal Science Report, Agricultural Experimental Station, Oklahoma State University, 121-124

Fletcher K. L., Topliff D. R., Cooper S. R., Freeman D. W., Geisert R. D. (2000) Influence of age and sex on serum osteocalcin concentrations in horses at weaning and during physical conditioning. J. Equine Vet. Sci. 20, 124-126

Fueller C. J., Barr A. R., Sharif M., Dieppe P. A. (2001) Cross-sectional comparison of synovial fluid biochemical markers in equine osteoarthritis and the correlation of these markers with articular cartilage damage. Osteoarth. Cartilage 9, 49-55

Henson F. M. D., Davies M. E., Skepper J. N., Jeffcott L. (1995) Localisation of alkaline phosphatase in equine growth cartilage. J. Anat. 187, 151-159

Hoyt S., Siciliano P. D. (1999) A comparison of ELISA and RIA techniques for the detection of serum osteocalcin in horses. Proceedings of the 16th Equine Nutrition and Physiology Symposium 16, 351-352

Jackson B. F., Lonnell C., Verheyen K., Wood J. L., Pfeiffert D. U., Price J. S. (2003a) Gender differences in bone turnover in 2-year-old Thoroughbreds. Equine Vet. J. 35, 702-706

Jackson B. F., Goodship A. E., Eastell R., Price J. S. (2003b) Evaluation of serum concentrations of biochemical markers of bone metabolism and insulin-like growth factor I associated with treadmill exercise in young horses. Am. J. Vet. Res. 64, 1549-1556

Jackson B. F., Blumsohn A., Goodship A. E., Wilson A. M., Price J. S. (2003c) Circadian variation in biochemical markers of bone cell activity and insulin-like growth factor-I in two-year-old horses. J. Anim. Sci. 11, 2804-2810

Jackson B. F., Reed S. R., Price J. S., Verheyen K. L. (2015) Relationship between serum biomarkers of cartilage and bone metabolism and joint injury in young Thoroughbred racehorses in training. Am. J. Vet. Res. 76, 679-687

Lepage O. M., Marcoux M., Tremblay A. (1990) Serum osteocalcin of bone Gla-protein, a biochemical marker for bone metabolism in horses: Differences in serum levels with age. Can. J. Vet. Res. 54, 223-226

Lepage O. M., Marcoux M., Tremblay A., Dumas G. (1992) Sex does not influence serum osteocalcin levels in Standardbred horses of different ages. Can. J. Vet. Res. 56, 379-381

Lepage O. M., Carstanjen B., Uebelhart D. (2001) Non-Invasive Assessment of Equine Bone: An Update. Vet. J. 161, 10-23

Pastoret V., Carstanien B., Lejeune J. P., Farnir F., Remy B., Reginster J. Y., Serteyn D., Gabriel A. (2007) Evaluation of serum osteocalcin and CTX-I in Ardenner horses with special reference to juvenile interphalangeal joint disease. J. Vet. Med. A Physiol. Pathol. Clin. Med. 54, 458-463

Price J. S., Jackson B., Eastell R., Goodship A. E., Blumsohn A., Wright I., Stoneham S., Lanyon L. E., Russell R. G. G. (1995) Age related changes in biochemical markers of bone metabolism in horses. Equine Vet. J. 3, 201-207

Price J. S., Jackson B. F., Gray J. A., Harris P. A., Wright I. M., Pfeiffer D. U., Robins S. P., Eastell R., Ricketts S. W. (2001) Biochemical markers of bone metabolism in growing thoroughbreds: a longitudinal study. Res. Vet. Sci. 71, 37-44

Risteli L., Risteli J. (1993) Biochemical markers of bone metabolism. Ann. Med. 25, 385-393

Roshan-Ghias A., Lambers F. M., Gholam-Rezaee M., Muller R., Pioletti D. P. (2011) In vivo loading increases mechanical properties of scaffold by affecting bone formation and bone resorption rates. Bone 49, 1357-1364

Saini P. K., Saini S. K. (1978) Origin of serum alkaline phosphatase in the dog. Am. J. Vet. Res. 39, 1510-1513

Seibel M. J. (2005) Biochemical Markers of Bone Turnover Part I: Biochemistry and Variability. Clin. Biochem. Rev. 26, 97-122

Vervuert I., Coenen M., Wedemeyer U., Harmeyer J. (2002) Biochemical markers of bone activity in young standardbred horses during different types of exercise and training. J. Vet. Med. A Physiol. Pathol. Clin. Med. 49, 396-402

Vervuert I., Lange A., Winkelsett S., Ellendorff F., Coenen M. (2005) Changes in bone markers and intact PTH in blood during a common training programme for three-day eventing horses. Pferdeheilkunde 21, 439-445 